\title{
Clinical Significance of the Decreased Expression of hsa_circ_001242 in Oral Squamous Cell Carcinoma
}

\author{
Shuai Sun, ${ }^{1,2}$ Bowen Li, ${ }^{1,2}$ Yufan Wang, ${ }^{2}$ Xiang Li, ${ }^{2}$ Panpan Wang, ${ }^{2}$ Feng Wang, \\ Wei Zhang ${ }^{3},^{3}$ and Hongyu Yang ${ }^{1,2}$ \\ ${ }^{1}$ Peking University Shenzhen Hospital Clinical College, Anhui Medical University, Hefei, Anhui, China \\ ${ }^{2}$ Department of Oral and Maxillofacial Surgery, Peking University Shenzhen Hospital, Shenzhen, Guangdong, China \\ ${ }^{3}$ Biomedical Research Institute, Shenzhen Peking University-The Hong Kong University of Science and Technology Medical Center, \\ Shenzhen, China
}

Correspondence should be addressed to Wei Zhang; zhangweispace@yeah.net and Hongyu Yang; hyyang192@hotmail.com

Received 21 January 2018; Accepted 9 May 2018; Published 4 July 2018

Academic Editor: Nelson Yee

Copyright (c) 2018 Shuai Sun et al. This is an open access article distributed under the Creative Commons Attribution License, which permits unrestricted use, distribution, and reproduction in any medium, provided the original work is properly cited.

Background. Circular RNAs (circRNAs) are a type of covalently closed loop structure of endogenous RNAs. Recent studies have shown that circular RNAs may play an important role in human cancer. However, there is limited information on the function of circRNA in oral squamous cell carcinoma (OSCC). Methods. Hsa_circ_001242 expression levels in 40 paired OSCC tissues and four OSCC cell lines were selected using real-time quantitative reverse transcription polymerase chain reaction (qRT-PCR). A receiver operating characteristic (ROC) curve was used to evaluate the diagnostic value of hsa_circ_001242 in OSCC. Results. Hsa_circ_001242 was significantly downregulated in OSCC tissues compared to paired adjacent normal tissues $(P<0.001)$. Hsa_circ_001242 expression levels were significantly downregulated in four OSCC cell lines (SCC-9, SCC-15, SCC25, and CAL-27) than in human normal oral keratinocyte (HOK) cell lines. Moreover, the expression level of hsa_circ_001242 was negatively correlated with tumor size and T stage $(P<0.05)$. The area under the ROC curve was 0.784 . Conclusion. This study showed that hsa_circ_001242 was significantly downregulated in OSCC and may act as a potential novel biomarker for the diagnosis and treatment of OSCC.

\section{Introduction}

Oral squamous cell carcinoma (OSCC) is the third most common cancer in developing countries and ranks sixth among systemic cancers worldwide $[1,2]$. Although there have been some improvements in the diagnosis and clinical treatment of OSCC, the 5-year overall survival rate of OSCC patients has not improved and remains $<50 \%$ over the last three decades [3, 4]. In addition, many patients have a poor response to therapy and high recurrence rates [5]. Therefore, it is important to determine effective molecular diagnostic markers and therapeutic targets of OSCC.

Circular RNAs (circRNAs) are a type of covalently closed loop structure of endogenous RNAs, which are characterized by linking the $3^{\prime}$ and $5^{\prime}$ ends generated by backsplicing. Unlike linear RNAs, circRNAs require high conservation, have high stability and tissue specificity, and are not easily degraded by enonuclease [6, 7]. In the 1970s, circRNAs were first discovered in Sendai viruses and then were clearly observed in eukaryotes $[8,9]$. CircRNAs were initially deemed to the products of aberrant RNA splicing, without attracting attention [10]. With the development of RNA high-throughput sequencing technology and bioinformatics, thousands of circRNAs have been discovered in the human genome [11]. Numerous researches have shown that circRNAs can be used as new biomarkers for clinical diagnosis and treatment of cancer [12-14]. However, there is little known about the role of circRNA in OSCC.

Searching OSCC associated circRNAs from circBase [15]. We first confirmed that hsa_circ_001242, which is located at chr10: 17157441-17168917 (Figure 1), was significantly downregulated in OSCC cell lines and OSCC tissues. 


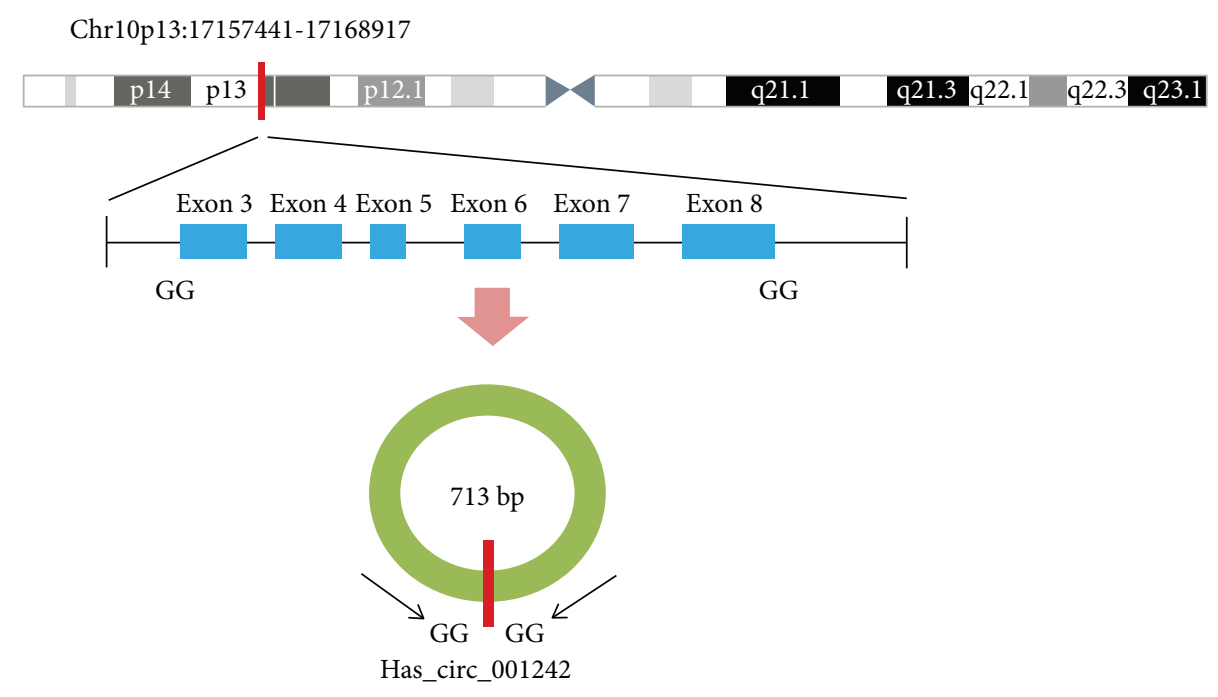

Figure 1: Hsa_circ_001242 is encoded from chromosomal region 10p13. Six exons of them form hsa_circ_001242 from exon 3 to exon 8.

Hsa_circ_001242, with a spliced sequence length of $713 \mathrm{bp}$, associated gene symbol is TRDMT1 (tRNA aspartic acid methyltransferase 1) and composed of six exons from exon 3 to exon 8. Its expression levels in tissues at various stages of OSCC were then explored, and the potential relationship between hsa_circ_001242 expression levels and patients' clinicopathological factors were investigated. A receiver operating characteristic (ROC) curve was constructed for evaluating the diagnostic value of hsa_circ_001242. Our data indicate that hsa_circ_001242 may be a potential biomarker for the diagnosis of OSCC.

\section{Materials and Methods}

2.1. Patients and Specimens. Forty oral cancer tissue samples and paired adjacent normal tissues were collected from the Department of Oral and Maxillofacial Surgery, Shenzhen Hospital, Peking University (Shenzhen, China), from December 2016 to May 2017. All samples were verified by histopathology. No patient previously underwent OSCC surgery, chemotherapy, or radiotherapy. Tissue samples were stored at $-80^{\circ} \mathrm{C}$ before use. This study was approved by Ethics Committee of Peking University Health Science Center (IRB00001053-08043).

2.2. RNA Extraction. Total RNA was extracted from OSCC tissues and paired adjacent normal tissues using the TRIzol reagent (Invitrogen, Carlsbad, CA, USA) according to the manufacturer's protocol. Total RNA from each specimen was quantified, and quality assurance was conducted using a NanoDrop ND2000 spectrophotometer (NanoDrop, Wilmington, DE, USA). Reaction mixture $(20 \mu \mathrm{l})$ containing $1 \mu \mathrm{g}$ total RNA was reverse transcribed to cDNA using PrimeScript RT-polymerase (Takara, Dalian, China).

The RNA sample was dissolved in RNase-free water. The yield and purity were measured using a NanoDrop 2000 instrument (Thermo Fisher Scientific, Waltham, MA, USA). The integrity of the RNA was determined using $1 \%$ formaldehyde denaturing gel electrophoresis. A PrimeScript
RT Reagent Kit (Takara Bio, Nojihigashi, Kusatsu, Japan) was used for the production of complementary DNA (cDNA) by reverse transcription, according to the manufacturer's instructions. qRT-PCR was performed using SYBR-Green Premix Ex Taq (Takara Bio, Nojihigashi, Kusatsu, Japan) and was monitored using the ABI PRISM 7500 Sequence Detection System (Applied Biosystems, Life Technologies, Waltham, MA, USA). The relative expression levels of circRNAs were determined by qRT-PCR. The sequences of the primers used in the qRT-PCR assay are shown in Supplementary Table 1.

The sequences of the hsa_circ_001242 primers were as follow: $5^{\prime}$-GCCCACTTGTAGAAGGTCCG-3' (forward primer) and reverse $5^{\prime}$-CTGGCAGGGAGGGCTCATTA-3' (reverse primer). The primer sequences for $\beta$-actin were $5^{\prime}$ AAACTGGAACGTTGAGAGTG-3' (forward primer) and 5'-AGTGGTCTGGCTTTTAGGT-3' (reverse primer). The reaction conditions were as follows: $95^{\circ} \mathrm{C}$ at $5 \mathrm{~min}$ for a preincubation and 40 cycles of $95^{\circ} \mathrm{C}$ for $5 \mathrm{~s}$, annealing temperature of $60^{\circ} \mathrm{C}$ for primer pairs for $30 \mathrm{~s}$, and $72^{\circ} \mathrm{C}$ for $20 \mathrm{~s}$. RNA levels were normalized using $\beta$-actin as the internal control.

2.3. Cell Culture. The human OSCC cell lines, SCC9, SCC15, SCC25, and CAL27, were obtained from the College of Stomatology, Wuhan University (Wuhan, China). Human oral keratinocytes (HOK) cells were obtained from the cell bank of the Chinese Academy of Sciences (Shanghai, China). SCC9 cells were cultured in DMED/F12 medium supplemented with $1 \%$ penicillin/streptomycin. SCC15, SCC25, CAL27, and HOK cells were cultured in Dulbecco's Modified Eagle Medium (DMED, GIBCO, China) supplemented with $1 \%$ penicillin/streptomycin (Life Technologies Inc., USA). All cells were cultured at $37^{\circ} \mathrm{C}$ under $5 \% \mathrm{CO}_{2}$.

2.4. Statistical Analysis. GraphPad Prism 5.0 Software (GraphPad Software, La Jolla, CA, USA) was used to analyze the obtained data. Results of hsa_circ_001242 expression for 


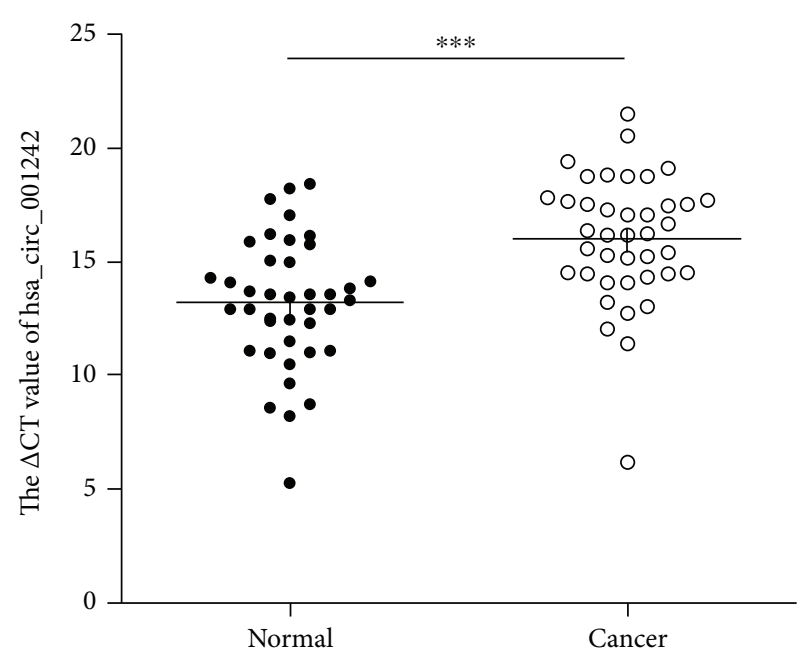

Figure 2: Hsa_circ_001242 expression levels in 40 pairs of OSCC tissues compared to that in paired adjacent normal tissues. Higher $\triangle \mathrm{Ct}$ value indicates lower expression. Data are expressed as mean $\pm \mathrm{SD} ;{ }^{* * *} P<0.001$.

OSCC tissues and paired adjacent normal tissues or between OSCC cell lines and HOK cell lines were compared using a paired $t$-test. A nonpaired $t$-test was used to analyze the relationship between hsa_circ_001242 expression level and clinicopathological factors in OSCC patients. The ROC curve was constructed to evaluate the diagnostic values. ${ }^{*} P<0.05,{ }^{* *} P<0.01$, and ${ }^{* * *} P<0.001$ were considered statistically significant.

\section{Results}

3.1. Expression of hsa_circ_001242 in OSCC Tissues. In this study, we first observed that the expression levels of hsa_circ_001242 were evidently downregulated in 40 OSCC tissue samples compared to that in paired adjacent normal tissues by qRT-PCR $(n=40, P<0.001)$ (Figure 2$)$.

3.2. Hsa_circ_001242 Expression Levels in OSCC Cell Lines. Furthermore, we explored the expression levels of hsa_circ_001242 in the HOK cell lines and four human OSCC cell lines, SCC-9, SCC-15, SCC-25, and CAL27. Expression levels in the OSCC cell lines were significantly lower than those in the HOK cell lines (Figure 3).

3.3. Relationship between hsa_circ_001242 Levels and Clinicopathological Factors. Clinicopathological analysis revealed that hsa_circ_001242 expression level was significantly associated with clinicopathological factors of OSCC patients. As presented in Table 1, hsa_circ_001242 expression levels were negatively related to tumor size $(P=0.0125)$ and T stage $(P=0.0434)$ but were not associated with other clinicopathological features, such as age, gender, tumor differentiation, TNM stage, or lymphatic metastasis $(P>0.05)$.

3.4. The Diagnostic Value of hsa_circ_001242 in OSCC. To estimate the diagnostic value of hsa_circ_001242 in OSCC, an ROC curve was constructed for differentiating OSCC

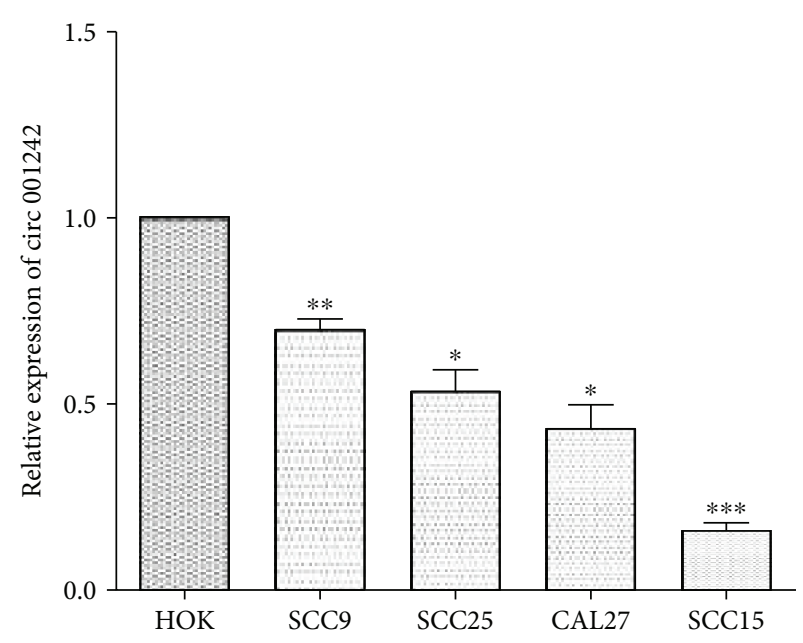

Figure 3: Hsa_circ_001242 expression in different OSCC cell lines. Hsa_circ_001242 expression levels in four OSCC cell lines (SCC-9, SCC-15, SCC-25, and CAL-27), and normal human oral keratinocyte cell lines were assessed by qRT-PCR. Data are presented as mean $\pm \mathrm{SD} ;{ }^{*} P<0.05,{ }^{* *} P<0.01$, and ${ }^{* * *} P<0.001$.

TABle 1: Primer sequences.

\begin{tabular}{lcc}
\hline Primer set & Forward primer & Reverse primer \\
\hline hsa_circ_001242 & GCCCACTTGTA & CTGGCAGGGAG \\
& GAAGGTCCG & GGCTCATTA \\
$\beta$-Actin & AAACTGGAACG & AGTGGTCTGGC \\
& TTGAGAGTG & TTTTAGGT \\
\hline
\end{tabular}

tissues from paired adjacent normal tissues. The area under the ROC curve (AUC) was 0.784 (95\% confidence interval $(C I)=0.717-0.867 ; P<0.001$; Figure $4(a))$. The cutoff value was 14.4 (Figure $4(\mathrm{~b})$ ), and the corresponding sensitivity and specificity of hsa_circ_001242 were $72.5 \%$ and $77.5 \%$, respectively. When the area under the curve is larger, the diagnostic value of the variable is higher. Values below the cutoff value are negative, while those exceeding the cutoff value are positive. Therefore, hsa_circ_001242 may act as a potential biomarker for the diagnosis of OSCC.

\section{Discussion}

CircRNAs are a class of noncoding RNAs (ncRNAs) that have been neglected as transcriptional noise in eukaryotes for the past 30 years $[10,16,17]$. Recent studies have found that some circRNAs serve as an important regulatory role and do not splice noise as previously thought [18]. Compelling evidence has manifested that circRNA can regulate gene expression through multiple mechanisms. CircRNAs act as a sponge of miRNA molecules and competitively bind miRNAs to regulate gene expression $[19,20]$. Exonintron circRNAs (EIciRNAs) can regulate transcription by RNA-RNA interactions in the nucleus [21, 22]. Some reports showed that circRNAs have the function of being translated into proteins [23, 24]. These studies indicate that the circular RNA plays an important role in transcriptional and posttranscriptional levels and can serve as an ideal marker for disease diagnosis. Previous studies 


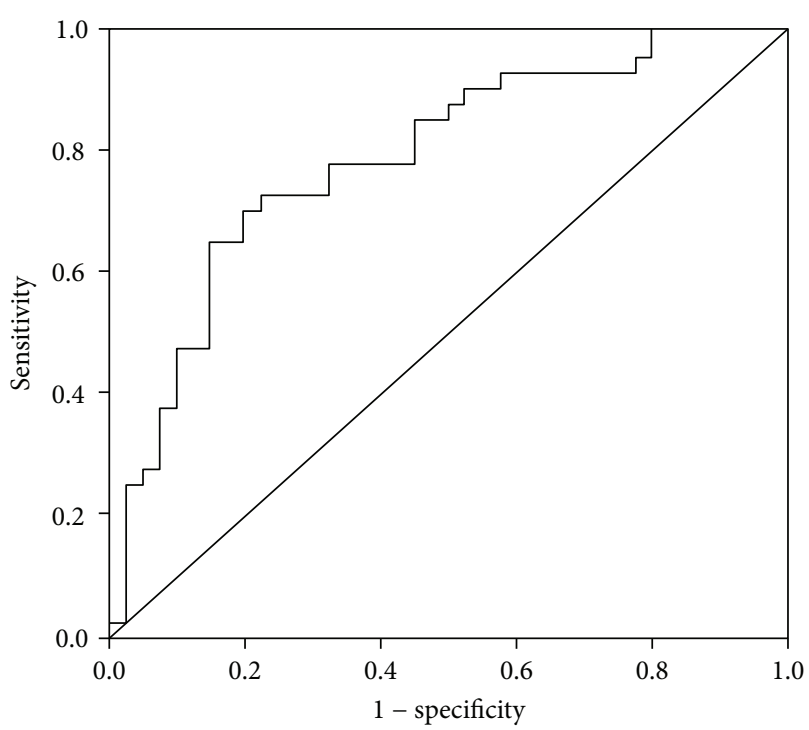

(a)

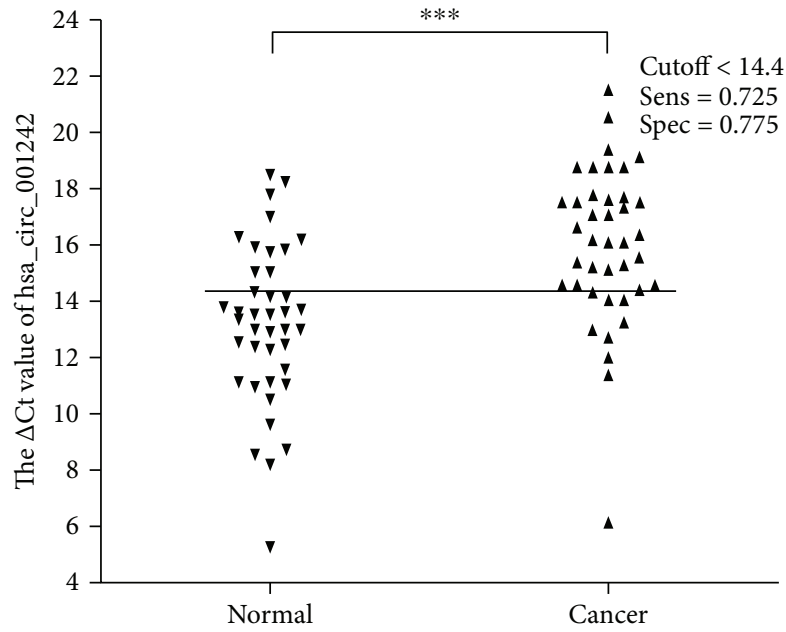

(b)

Figure 4: The diagnostic value of hsa_circ_001242 in OSCC. (a) The area under the ROC curve (AUC) was $0.784(95 \%$ CI $=0.683-0.885$, $P<0.001$ ), (b) and the cutoff of hsa_circ_001242 was 14.4 . The sensitivity and specificity were 0.725 and 0.775 , respectively. Data are presented as mean $\pm \mathrm{SD} ;{ }^{* * *} P<0.001$.

have suggested that circRNAs may play an important role in the development of cancer and act as potential biomarkers for cancer $[25,26]$. For example, Li Wan et al. [27] have revealed that circ-ITCH is overexpressed in lung cancer tissues and inhibits the $\mathrm{Wnt} / \beta$-catenin signaling pathway by acting as a sponge for miR-7 and miR-214. Zhu et al. [28] have reported that circ-BANP expression in colorectal cancerous tissues is significantly upregulated than those in adjacent normal tissues. Huang et al. [29] have observed that hsa_circ_0000745 is downregulated in gastric cancer tissues as well as in the plasma samples from patients with gastric cancer. Their study revealed that the circular RNA hsa_circ_0000745 may serve as a diagnostic marker for gastric cancer. Yao et al. [30] have confirmed that circZKSCAN1 is downregulated in hepatocellular carcinoma and may act as a diagnostic biomarker. Thus, we believe that circRNAs may serve as novel molecular markers for cancer.

In this study, we first observed that hsa_circ_001242 was downregulated in both OSCC tissues and OSCC cell lines. Furthermore, the study on the clinical features and the expression of hsa_circ_001242 indicated that hsa_circ_001242 expression level was negatively relevant to tumor size and $\mathrm{T}$ stage (Table 2). These data suggest that circRNAs may play a role in carcinogenesis and progress of OSCC. The ROC curve was constructed for differentiating OSCC tissues from the controls. Our results demonstrated that AUC was 0.784 . The sensitivity and specificity of hsa_circ_001242 were 0.725 and 0.775 , respectively. Furthermore, we observed that the cutoff value of hsa_circ_001242 was 14.4. To further understand the biological role of circRNAs in OSCC, both in vitro and in vivo assays should be performed in the future after successfully engineering of circRNAs.

In conclusion, our study manifested that hsa_circ_001242 was significantly downregulated in OSCC tissues and OSCC cell lines. In addition, the expression of hsa_circ_001242
TABle 2: Correlation between clinicopathological factors and hsa_circ_001242 expression levels $(\Delta \mathrm{Ct})$ in oral squamous cell carcinoma patients.

\begin{tabular}{|c|c|c|c|}
\hline Characteristics & $\begin{array}{c}\text { Number of } \\
\text { patients }\end{array}$ & Mean \pm SD & $P$ value \\
\hline \multicolumn{4}{|l|}{ Age (year) } \\
\hline$\geq 60$ & 14 & $16.88 \pm 0.644$ & \multirow{2}{*}{0.145} \\
\hline$<60$ & 26 & $15.52 \pm 0.572$ & \\
\hline \multicolumn{4}{|l|}{ Gender } \\
\hline Male & 28 & $16.05 \pm 0.537$ & \multirow{2}{*}{0.871} \\
\hline Female & 12 & $15.89 \pm 0.813$ & \\
\hline \multicolumn{4}{|l|}{ Tumor size $(\mathrm{cm})$} \\
\hline$\geq 5$ & 6 & $13.43 \pm 0.543$ & \multirow{2}{*}{$0.0125^{*}$} \\
\hline$<5$ & 34 & $16.45 \pm 0.408$ & \\
\hline \multicolumn{4}{|l|}{ Differentiation } \\
\hline Well and moderate & 34 & $16.23 \pm 0.424$ & \multirow{2}{*}{0.221} \\
\hline Poor & 6 & $14.70 \pm 0.747$ & \\
\hline \multicolumn{4}{|l|}{ T stage } \\
\hline $\mathrm{T} 1-2$ & 29 & $16.58 \pm 0.45$ & \multirow{2}{*}{$0.0317^{*}$} \\
\hline T3-4 & 11 & $14.47 \pm 0.99$ & \\
\hline \multicolumn{4}{|l|}{ TNM } \\
\hline I and II & 19 & $16.76 \pm 0.61$ & \multirow{2}{*}{0.103} \\
\hline III and IV & 21 & $15.31 \pm 0.61$ & \\
\hline \multicolumn{4}{|l|}{ Lymphatic metastasis } \\
\hline No & 24 & $16.50 \pm 0.516$ & 0.170 \\
\hline $\mathrm{N} 1-3$ & 16 & $15.25 \pm 0.773$ & \\
\hline
\end{tabular}

* indicated statistical significance.

expression is negatively correlated with tumor size and $\mathrm{T}$ stage. Thus, hsa_circ_001242 may serve as a potential diagnostic biomarker for OSCC. 


\section{Data Availability}

The data used to support the findings of this study are available from the corresponding author upon request.

\section{Conflicts of Interest}

The authors declare that there is no conflict of interest regarding the publication of this article.

\section{Authors' Contributions}

Shuai Sun and Bowen Li contributed equally to this work.

\section{Acknowledgments}

This study was supported by the National Natural Science Foundation of China (Grant no. 81572654), the Basic Research Program of Shenzhen Innovation Council of China (Grant nos. JCYJ20150403091443303, JCYJ201504030914 43286, JCYJ201604287 3933559, and SZBC2017023), and Sanming Project of Medicine in Shenzhen (SZSM 20151 2036, Oral and Maxillofacial Surgery Team, Professor Yu Guangyan, Stomatology Hospital Peking University).

\section{Supplementary Materials}

Supplementary Information Table 1. Detailed information on clinical and oral squamous cell carcinoma characteristics of patients and the information of metastasis. (Supplementary Materials)

\section{References}

[1] M. S. Rosebush, S. K. Rao, S. Samant et al., "Oral cancer: enduring characteristics and emerging trends," The Journal of the Tennessee Dental Association, vol. 91, no. 2, pp. 24-27, 2012.

[2] J. Casiglia and S. B. Woo, "A comprehensive review of oral cancer," General Dentistry, vol. 49, no. 1, pp. 72-82, 2001.

[3] P. R. Brocklehurst, S. R. Baker, and P. M Speight, "Oral cancer screening: what have we learnt and what is there still to achieve?," Future Oncology, vol. 6, no. 2, pp. 299-304, 2010.

[4] R. Siegel, J. Ma, Z. Zou, and A. Jemal, "Cancer statistics, 2014," CA: a Cancer Journal for Clinicians, vol. 64, no. 1, pp. 9-29, 2014.

[5] D. Wangsa, M. Ryott, E. Åvall-Lundqvist et al., "Ki-67 expression predicts locoregional recurrence in stage I oral tongue carcinoma," British Journal of Cancer, vol. 99, no. 7, pp. 1121-1128, 2008.

[6] L.-L. Chen and L. Yang, "Regulation of circRNA biogenesis," RNA Biology, vol. 12, no. 4, pp. 381-388, 2015.

[7] H. Suzuki, Y. Zuo, J. Wang, M. Q. Zhang, A. Malhotra, and A. Mayeda, "Characterization of RNase R-digested cellular RNA source that consists of lariat and circular RNAs from pre-mRNA splicing," Nucleic Acids Research, vol. 34, no. 8, article e63, 2006.

[8] D. Kolakofsky, "Isolation and characterization of Sendai virus DI-RNAs,” Cell, vol. 8, no. 4, pp. 547-555, 1976.

[9] M.-T. Hsu and M. Coca-Prados, "Electron microscopic evidence for the circular form of RNA in the cytoplasm of eukaryotic cells," Nature, vol. 280, no. 5720, pp. 339-340, 1979.

[10] C. Cocquerelle, B. Mascrez, D. Hétuin, and B. Bailleul, "Missplicing yields circular RNA molecules," The FASEB Journal, vol. 7, no. 1, pp. 155-160, 1993.

[11] S. Xia, J. Feng, L. Lei et al., "Comprehensive characterization of tissue-specific circular RNAs in the human and mouse genomes," Briefings in Bioinformatics, vol. 18, no. 6, pp. 984992, 2016.

[12] P. Li, S. Chen, H. Chen et al., "Using circular RNA as a novel type of biomarker in the screening of gastric cancer," Clinica Chimica Acta, vol. 444, pp. 132-136, 2015.

[13] L. Szabo and J. Salzman, "Detecting circular RNAs: bioinformatic and experimental challenges," Nature Reviews Genetics, vol. 17, no. 11, pp. 679-692, 2016.

[14] D. Rong, W. Tang, Z. Li et al., "Novel insights into circular RNAs in clinical application of carcinomas," OncoTargets and Therapy, vol. 10, pp. 2183-2188, 2017.

[15] “Circbase," http://circbase.org/cgi-bin/simplesearch.cgi.

[16] A. Kos, R. Dijkema, A. C. Arnberg, P. H. Van der Meide, and H. Schellekens, "The hepatitis delta $(\delta)$ virus possesses a circular RNA,” Nature, vol. 323, no. 6088, pp. 558-560, 1986.

[17] P. G. Zaphiropoulos, "Circular RNAs from transcripts of the rat cytochrome P450 2C24 gene: correlation with exon skipping," Proceedings of the National Academy of Sciences of the United States of America, vol. 93, no. 13, pp. 6536-6541, 1996.

[18] L. Chen, C. Huang, X. Wang, and G. Shan, "Circular RNAs in eukaryotic cells,” Current Genomics, vol. 16, no. 5, pp. 312318, 2015.

[19] F. A. Karreth, Y. Tay, D. Perna et al., "In vivo identification of tumor- suppressive PTEN ceRNAs in an oncogenic BRAFinduced mouse model of melanoma," Cell, vol. 147, no. 2, pp. 382-395, 2011.

[20] T. B. Hansen, J. Kjems, and C. K. Damgaard, "Circular RNA and miR-7 in cancer," Cancer Research, vol. 73, no. 18, pp. 5609-5612, 2013.

[21] Z. Y. Li, C. Huang, C. Bao et al., "Exon-intron circular RNAs regulate transcription in the nucleus," Nature Structural of Molecular Biology, vol. 22, no. 3, pp. 256-264, 2015.

[22] C. Huang and G. Shan, "What happens at or after transcription: insights into circRNA biogenesis and function," Transcription, vol. 6, no. 4, pp. 61-64, 2015.

[23] N. Abe, K. Matsumoto, M. Nishihara et al., "Rolling circle translation of circular RNA in living human cells," Scientific Reports, vol. 5, no. 1, article 16435, 2015.

[24] Y. Wang and Z. Wang, "Efficient backsplicing produces translatable circular mRNAs," RNA, vol. 21, no. 2, pp. 172-179, 2015.

[25] Y. Chen, C. Li, C. Tan, and X. Liu, "Circular RNAs: a new frontier in the study of human diseases," Journal of Medical Genetics, vol. 53, no. 6, pp. 359-365, 2016.

[26] S. Meng, H. Zhou, Z. Feng et al., "CircRNA: functions and properties of a novel potential biomarker for cancer," Molecular Cancer, vol. 16, no. 1, p. 94, 2017.

[27] L. Wan, L. Zhang, K. Fan, Z. X. Cheng, Q. C. Sun, and J. J. Wang, "Circular RNA-ITCH suppresses lung cancer proliferation via inhibiting the Wnt/ $\beta$-catenin pathway," BioMed Research International, vol. 2016, Article ID 1579490, 11 pages, 2016.

[28] M. Zhu, Y. Xu, Y. Chen, and F. Yan, "Circular BANP, an upregulated circular RNA that modulates cell proliferation in 
colorectal cancer," Biomedicine \& Pharmacotherapy, vol. 88, pp. 138-144, 2017.

[29] M. Huang, Y.-R. He, L.-C. Liang, Q. Huang, and Z.-Q. Zhu, "Circular RNA hsa_circ_0000745 may serve as a diagnostic marker for gastric cancer," World Journal of Gastroenterology, vol. 23, no. 34, pp. 6330-6338, 2017.

[30] Z. Yao, J. Luo, K. Hu et al., "ZKSCAN1 gene and its related circular RNA (circZKSCAN1) both inhibit hepatocellular carcinoma cell growth, migration, and invasion but through different signaling pathways," Molecular Oncology, vol. 11, no. 4, pp. 422-437, 2017. 


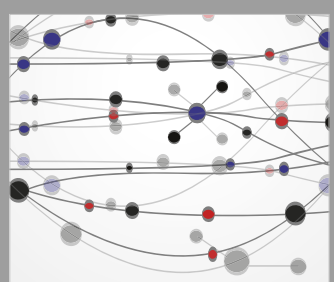

The Scientific World Journal
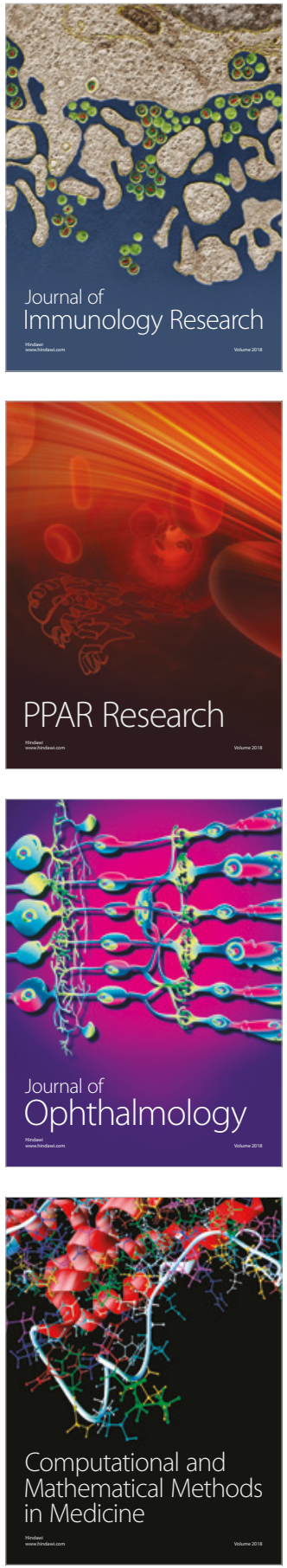

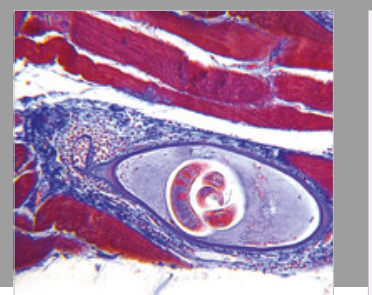

Gastroenterology Research and Practice

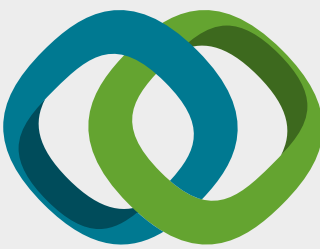

\section{Hindawi}

Submit your manuscripts at

www.hindawi.com
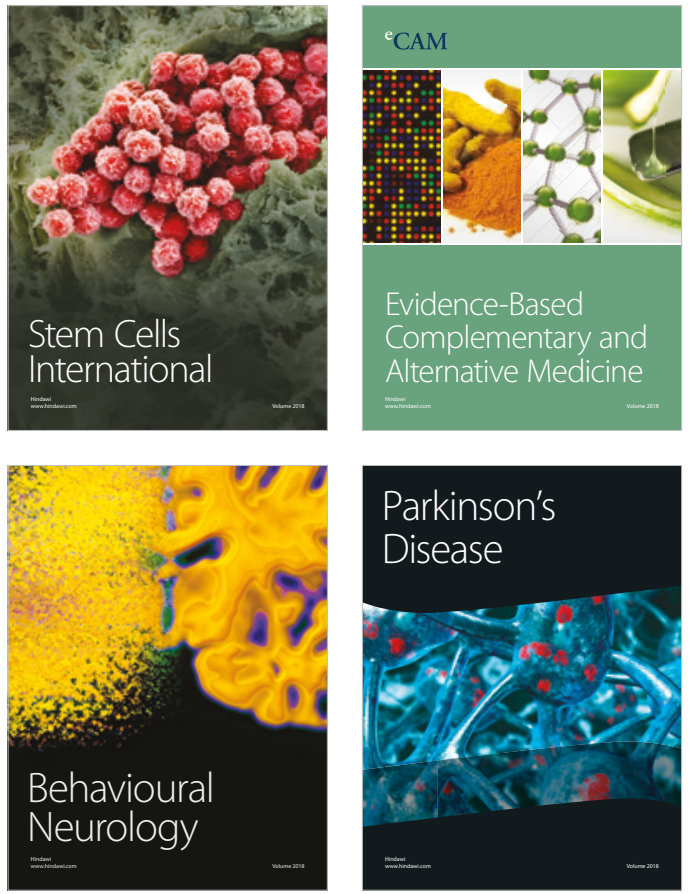

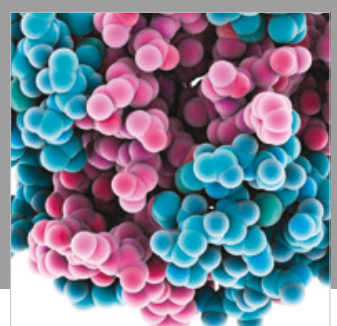

ournal of

Diabetes Research

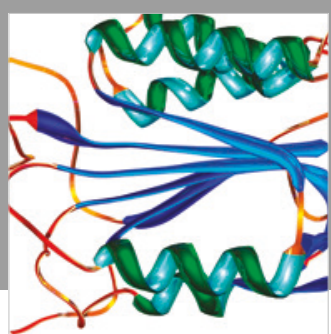

Disease Markers
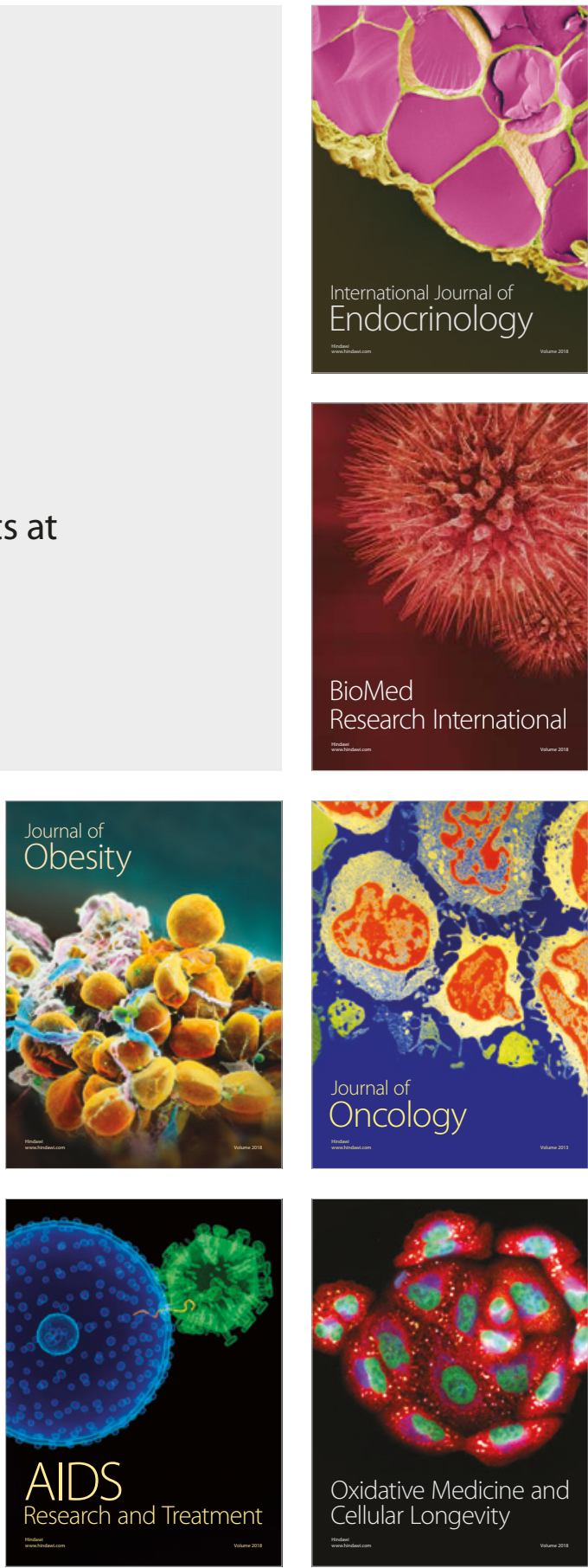